\title{
Metastatic Parotid Gland Carcinoma With ERBB2 Amplification With Complete Response to Fam-Trastuzumab Deruxtecan
}

\author{
Navika D. Shukla, BA ${ }^{1}$; Ryan S. Chiang, MD²; and Alexander D. Colevas, MD $^{3}$
}

\begin{abstract}
HER2 mutations have been shown to be targetable in select cases of salivary gland cancers with overexpression or amplification of the HER2 oncogene. Fam-trastuzumab deruxtecan, a novel antibodydrug conjugate that combines trastuzumab with a topoisomerase I inhibitor, has recently demonstrated efficacy as a third-line agent in HER2-overexpressing breast cancer after trastuzumab failure. These promising results in breast cancer suggest a potential paradigm for use in other tumors with known HER2 alterations, including salivary gland cancer. This report describes a 67-year-old man with HER2positive metastatic parotid gland carcinoma who experienced disease progression after parotidectomy with adjuvant cisplatin-based chemoradiation, neratinib, and ado-trastuzumab emtansine. After disease progression on the latter HER2-directed therapy, his malignancy demonstrated complete response to fam-trastuzumab deruxtecan, which has been sustained for the past 7 months. Famtrastuzumab deruxtecan appears to be a well-tolerated therapeutic option in patients with HER2-positive salivary gland carcinoma, with activity demonstrated after progression on ado-trastuzumab emtansine and HER2-directed kinase inhibition. Further studies should be conducted to explore the use of this agent in HER2-positive salivary gland cancers.
\end{abstract}

J Natl Compr Canc Netw 2022;20(2):102-104 doi: 10.6004/jnccn.2021.7089

\footnotetext{
${ }^{1}$ Stanford University School of Medicine; and ${ }^{2}$ Department of Medicine, and ${ }^{3}$ Division of Medical Oncology, Department of Medicine, Stanford University Medical Center, Stanford, California.
}

HER2 is a member of the epidermal growth factor receptor (EGFR) family overexpressed in several malignancies. Although typically used in HER2-positive gastric and breast cancers, several studies have shown a role for HER2-targeted therapies in other cancer settings. This includes salivary duct carcinomas (SDCs), which have been commonly linked to the overexpression or amplification of HER2 in gene profiling studies. ${ }^{1-7}$ More recently, newer second- and third-generation anti-HER2 agents have emerged, including ado-trastuzumab emtansine (T-DM1) and fam-trastuzumab deruxtecan. In breast and gastric cancer, repeated targeting of the HER2 receptor with these newer agents has demonstrated promise as an effective treatment strategy, suggesting a potential role for these agents in the resistant setting in salivary gland cancer as well. ${ }^{8,9}$ Although the literature thus far demonstrates that both trastuzumab emtansine and trastuzumab deruxtecan alone hold promise in salivary gland cancers, there have been no studies to date evaluating trastuzumab deruxtecan after trastuzumab emtansine failure in this population..$^{10-12}$

This report describes a patient who presented with high-grade stage IV SDC arising from a pleomorphic adenoma of the left parotid gland with HER2 amplification. Our patient experienced disease progression despite initial response to multiple lines of therapy, including adjuvant chemoradiation with cisplatin; neratinib, a dual inhibitor of the HER2 and EGFR kinases; and T-DM1, an antibody-drug conjugate of an HER2-directed monoclonal antibody and microtubule poison, before achieving a sustained complete response on fam-trastuzumab deruxtecan.

\section{Case Presentation}

A 67-year-old White man presented with left-sided ear discomfort and facial paresis. MRI revealed the presence of a 3-cm left parotid mass without lymphadenopathy. A core needle biopsy was performed, and pathology revealed adenocarcinoma with perineural invasion. He underwent parotidectomy with findings consistent with T4aN0 SDC ex pleomorphic adenoma, and he completed adjuvant chemoradiation with cisplatin for 6 weeks. Nine months later, he presented with worsening back pain. 
Imaging showed a new T8 compression fracture concerning for metastatic disease and impending cord compression. He underwent T5 to T10 posterior spinal fusion with T8 costotransversectomy. Consistent with the original primary parotid neoplasm, surgical pathology confirmed metastatic adenocarcinoma that was androgen receptor (AR)-positive by immunohistochemistry and ERBB2 (HER2)-amplified, DNMT3A-mutated, and microsatellite stable by FoundationOne genetic testing. Additional HER2 staining by immunohistochemistry showed strong circumferential membrane staining in $>10 \%$ of the SDC cells and was scored as $3+$. PET imaging revealed diffuse osseous metastatic disease throughout the cervical and thoracic spine, left scapula, ribs, and femoral head. The patient underwent additional stereotactic body radiotherapy to the thoracic spine and was started on bone-directed therapy with denosumab. He received no AR-directed therapy. Given his known HER2 amplification, he was eligible for neratinib under the SUMMIT clinical trial (ClinicalTrials.gov identifier: NCT01953926). Despite initial complete response, treatment was subsequently discontinued 7 months after initiation when interval PET imaging demonstrated an increased size and number of bilateral pulmonary nodules. Given disease progression, he was transitioned to T-DM1. Follow-up PET imaging 5 months after starting T-DM1 showed a radiologic complete response. After 10 months of T-DM1, repeat PET revealed increased disease burden of pulmonary nodules and bony disease. He was initiated on famtrastuzumab deruxtecan, with radiologic complete response seen after 2 months (Figure 1). At the time of writing, serial imaging for the past 7 months has

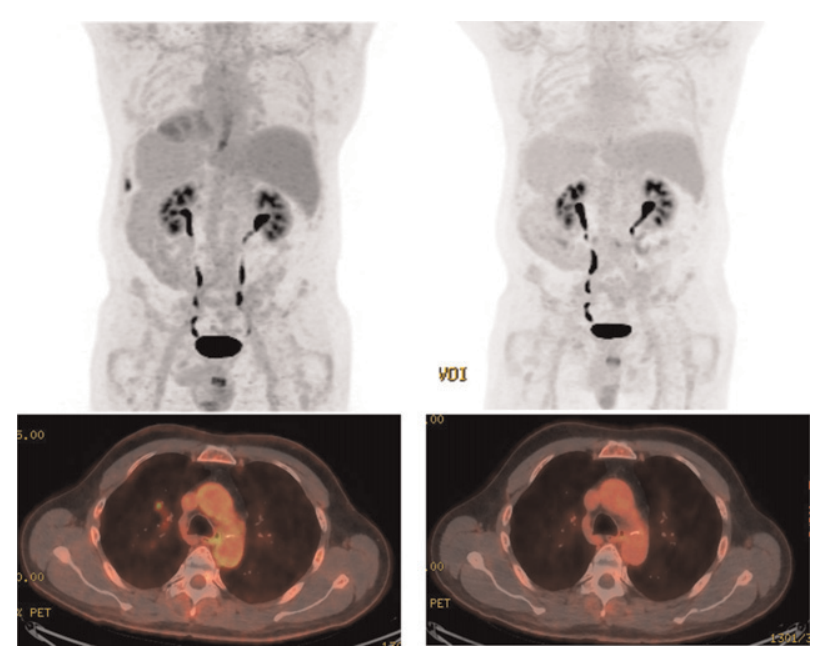

Figure 1. Progression of metastatic disease on ado-trastuzumab emtansine (right) followed by complete resolution of FDG-avid pulmonary nodules after 3 months of treatment with fam-trastuzumab deruxtecan (left). not shown any evidence of recurrence or metastatic disease, and he continues on surveillance imaging every 4 months. He has tolerated the drug well; adverse effects from infusion included transient fatigue and grade 1 nausea, vomiting, and gastroparesis.

\section{Discussion}

This report describes a patient with metastatic carcinoma ex pleomorphic adenoma with HER2 amplification who demonstrated a complete response after receiving famtrastuzumab deruxtecan despite disease progression on neratinib and T-DM1. To our knowledge, there has not been a similar case reported.

Pharmacologically, trastuzumab deruxtecan is an antibody-drug conjugate composed of an anti-HER2 antibody, a peptide linker, and a topoisomerase I inhibitor. Structurally, the drug is optimized for improved efficacy even in the setting of low HER2 expression when compared with previous anti-HER2 agents. ${ }^{13}$ It is generally well tolerated, although notable toxic effects include myelosuppression and interstitial lung disease. ${ }^{8,9}$

The reported prevalence of HER2 overexpression in salivary gland cancers is $7 \%$ to $56 \%$ and has been shown to vary by histologic subtype, with a higher prevalence in salivary duct subtypes, highlighting the utility of molecular profiling in treatment determination. ${ }^{1}$ The NCI-MATCH trial assessed the efficacy of T-DM1 in HER2-amplified nonbreast and nongastroesophageal malignancies and found evidence of durable partial response in salivary gland tumors. ${ }^{12}$ In a larger phase II basket trial, a cohort of 10 patients with HER2-amplified salivary gland tumors were treated with T-DM1. ${ }^{11}$ The overall response rate was $90 \%$, with $50 \%$ of patients demonstrating a complete response. Few studies to date, however, have evaluated trastuzumab deruxtecan in salivary gland cancer. Preliminary results of evaluating the agent in a heterogeneous cohort of solid tumors that included 8 salivary gland cancers have shown HER-amplified salivary gland tumors to be responsive to trastuzumab deruxtecan, but further studies are needed to better understand this effect. The complete response demonstrated by our patient on fam-trastuzumab deruxtecan despite disease progression while receiving neratinib and T-DM1 indicates a potential role for this drug in recurrent HER2-amplified salivary gland carcinoma cases that have progressed on prior lines of HER2-directed therapy.

In breast and gastric cancer settings, fam-trastuzumab deruxtecan has shown promise in patients who have experienced disease progression on prior therapy. Per the DESTINY-Breast01 trial, trastuzumab deruxtecan demonstrated durable antitumor activity in patients with HER2-positive metastatic breast cancer who had already received trastuzumab emantasine. ${ }^{9}$ Likewise, the 
DESTINY-Gastric01 trial selected patients with advanced gastric or gastroesophageal junction adenocarcinoma who had experienced progression on $\geq 2$ prior lines of therapy, at least 1 of which included an anti-HER2 agent, and randomized them to receive either trastuzumab deruxtecan or their physician's choice of chemotherapy. ${ }^{8}$ Fam-trastuzumab deruxtecan was associated with an increased objective response rate and improved overall survival. ${ }^{8}$ Response to this agent also suggests that resistance to prior therapy likely occurred through mechanisms other than HER2 alteration or downregulation. Perhaps resistance to these other agents occurred through increased expression of substrate-specific drug efflux transporters or, in the case of resistance to T-DM1, through mitotic catastrophe escape. ${ }^{14}$ Further studies are needed to elucidate mechanisms of resistance.

Other than HER2-directed therapy, another potential therapeutic option for many cases of recurrent or metastatic SDC includes androgen deprivation therapy (ADT) ${ }^{15-17}$ Although standardized guidelines for ADT in this setting have not yet been established, several studies have demonstrated excellent response with abiraterone, single-agent bicalutamide, or a combination of triptore- lin and bicalutamide. However, with no data yet available to support ADT versus HER2-directed therapy as an initial treatment strategy, this remains an area for further exploration.

\section{Conclusions}

This case report shows a complete response to trastuzumab deruxtecan with limited toxicity in the setting of recurrent, metastatic salivary gland cancer. This study provides anecdotal evidence that recurrent engagement of HER2-targeted therapies may have utility as a treatment pathway in salivary gland cancer similar to the model currently used in HER2-amplified breast and gastric cancer.

Submitted April 8, 2021; final revision received September 8, 2021; accepted for publication September 9, 2021

Disclosures: The authors have disclosed that they have not received any financial considerations from any person or organization to support the preparation, analysis, results, or discussion of this article.

Correspondence: Ryan S. Chiang, MD, Department of Medicine, Stanford University Medical Center, Stanford Hospital and Clinics, 300 Pasteur Drive Stanford, CA 94305. Email: rschiang@stanford.edu

\section{References}

1. Haddad R, Colevas AD, Krane JF, et al. Herceptin in patients with advanced or metastatic salivary gland carcinomas. A phase II study. Oral Oncol 2003;39:724-727

2. Kurzrock R, Bowles DW, Kang H, et al. Targeted therapy for advanced salivary gland carcinoma based on molecular profiling: results from MyPathway, a phase Ila multiple basket study. Ann Oncol 2020;31: 412-421.

3. Uijen MJM, Lassche G, van Engen-van Grunsven ACH, et al. Systemic therapy in the management of recurrent or metastatic salivary duct carcinoma: a systematic review. Cancer Treat Rev 2020;89:102069.

4. Glisson B, Colevas AD, Haddad R, et al. HER2 expression in salivary gland carcinomas: dependence on histological subtype. Clin Cancer Res 2004;10:944-946.

5. Palsgrove D, Allahabadi S, Khan SA. Genomic analysis of salivary gland cancer and treatment of salivary gland cancers. Surg Pathol Clin 2021;14 151-163.

6. Locati LD, Perrone $\mathrm{F}$, Losa $\mathrm{M}$, et al. Treatment relevant target immunophenotyping of 139 salivary gland carcinomas (SGCs). Oral Oncol 2009; 45:986-990.

7. Dalin MG, Desrichard A, Katabi N, et al. Comprehensive molecular characterization of salivary duct carcinoma reveals actionable targets and similarity to apocrine breast cancer. Clin Cancer Res 2016;22:4623-4633.

8. Shitara K, Iwata H, Takahashi S, et al. Trastuzumab deruxtecan (DS8201a) in patients with advanced HER2-positive gastric cancer: a doseexpansion, phase 1 study. Lancet Oncol 2019;20:827-836.

9. Modi S, Saura C, Yamashita T, et al. Trastuzumab deruxtecan in previously treated HER2-positive breast cancer. N Engl J Med 2020;382:610-621.
10. Tsurutani J, Iwata H, Krop I, et al. Targeting HER2 with trastuzumab deruxtecan: a dose-expansion, phase I study in multiple advanced solid tumors. Cancer Discov 2020;10:688-701.

11. Li BT, Shen R, Offin M, et al. Ado-trastuzumab emtansine in patients with HER2 amplified salivary gland cancers (SGCs): results from a phase II bas ket trial [abstract]. J Clin Oncol 2019;37(Suppl):Abstract 6001.

12. Jhaveri $\mathrm{KL}$, Wang $\mathrm{XV}$, Makker $\mathrm{V}$, et al. Ado-trastuzumab emtansine (T-DM1) in patients with HER2-amplified tumors excluding breast and gastric/gastroesophageal junction (GEJ) adenocarcinomas: results from the $\mathrm{NCl}-\mathrm{MATCH}$ trial (EAY131) subprotocol Q. Ann Oncol 2019;30:1821-1830.

13. Takegawa N, Nonagase $Y$, Yonesaka K, et al. DS-8201a, a new HER2-targeting antibody-drug conjugate incorporating a novel DNA topoisomerase I inhibitor, overcomes HER2-positive gastric cancer T-DM1 resistance. Int J Cancer 2017;141:1682-1689.

14. Hunter FW, Barker HR, Lipert B, et al. Mechanisms of resistance to trastuzumab emtansine (T-DM1) in HER2-positive breast cancer. Br J Cancer 2020;122:603-612

15. Boon E, van Boxtel W, Buter J, et al. Androgen deprivation therapy for androgen receptor-positive advanced salivary duct carcinoma: a nationwide case series of 35 patients in The Netherlands. Head Neck 2018;40: 605-613.

16. Locati LD, Perrone F, Cortelazzi B, et al. Clinical activity of androgen deprivation therapy in patients with metastatic/relapsed androgen receptorpositive salivary gland cancers. Head Neck 2016;38:724-731.

17. Urban D, Rischin D, Angel C, et al. Abiraterone in metastatic salivary duct carcinoma. J Natl Compr Canc Netw 2015;13:288-290. 